\title{
Collimating, relativistic, magnetic jets from rotating disks
}

\section{The axisymmetric field structure of relativistic jets and the example of the $M 87$ jet}

\begin{abstract}
C. Fendt and E. Memola
Astrophysikalisches Institut Potsdam, An der Sternwarte 16, 14482 Potsdam, Germany

e-mail: cfendt@aip.de; ememola@aip.de

Received 25 April 2000 / Accepted 19 October 2000

Abstract. We investigate the axisymmetric structure of collimating, relativistic, strongly magnetized (force-free) jets. In particular, we include the differential rotation of the foot points of the field lines in our treatment. The magnetic flux distribution is determined by the solution of the Grad-Shafranov equation and the regularity condition along the light surface. With differential rotation, i.e. the variation of the iso-rotation parameter $\Omega_{\mathrm{F}}$, the shape of the light surface is not known a priori and must be calculated in an iterative way. For the first time, we have calculated the force-free magnetic structure of truly two-dimensional, relativistic jets, anchored in a differentially rotating disk. Such an approach allows for a direct connection between parameters of the central source (mass, rotation) and the extension of the radio jet. In particular, this can provide a direct scaling of the location of the asymptotic jet light cylinder in terms of the central mass and the accretion disk magnetic flux distribution. We demonstrate that differentially rotating jets must be collimated to a smaller radius in terms of the light cylinder if compared to jets with rigid rotation. Also, the opening angle is smaller. Further we present an analytical estimate for the jet opening angle along the asymptotic branches of the light surface. In general, differential rotation of the iso-rotation parameter leads to an increase of the jet opening angle. Our results are applicable for highly magnetized, highly collimated, relativistic jets from active galactic nuclei and Galactic superluminal jet sources. Comparison to the M 87 jet shows agreement in the collimation distance. We derive a light cylinder radius of the M 87 jet of 50 Schwarzschild radii.
\end{abstract}

Key words. accretion, accretion disks - MHD - methods: numerical - ISM: jets and outflows - galaxies: individual: M 87 - galaxies: jets

\section{Formation of magnetic jets}

Observations of astrophysical jet sources have now established the idea that jet formation is always connected to the presence of an accretion disk and strong magnetic fields. This holds for various scales of energy output, jet velocity and nature of the jet emitting objects. Examples are jets from active galactic nuclei (AGN), Galactic superluminal jet sources, the example of a mildly relativistic jet from a neutron star (SS 433) and the numerous class of protostellar jets (see Zensus et al. 1995; Mirabel \& Rodriguez 1995; Mundt et al. 1990; Ray et al. 1996). Magnetic jets are believed to originate very close to the central object in the interaction region with the accretion disk. Beside observational arguments also theoretical considerations have shown that magnetic fields play an important role in jet formation and propagation (Blandford \& Payne 1982; Pudritz \& Norman 1983;

Send offprint requests to: C. Fendt
Shibata \& Uchida 1985; Sakurai 1985; Camenzind 1987; Lovelace et al. 1991).

If the central object is a black hole as it is the case for AGN and Galactic superluminal jet sources, the surrounding accretion disk is the only possible location for a field generation (by dynamo action or/and advection of flux). In the case of stellar objects (protostars, white dwarfs or neutron stars), the central star also carries a relatively strong magnetic field and it is not yet clear, whether the jet magnetosphere originates in the disk or in the star. However, a strong interaction between stellar field and accretion flow is evident. The jet formation process itself is not yet fully understood theoretically. In particular, for the mass transfer from the disk into the jet and the process of magnetic field generation a complete physical model is missing.

However, over the last decades the basic ideas of Blandford \& Payne (1982) have been extended by various authors. The general picture is the following. Matter 
is lifted from the disk into the magnetosphere and becomes magnetically accelerated (Ferreira 1997). Toroidal magnetic fields, generated by inertial back-reaction of the plasma on the poloidal field, may collimate the disk magnetosphere into a highly collimated jet flow (Camenzind 1987; Chiueh et al. 1991; Lovelace et al. 1991). In general, due to the complexity of the MHD equations, stationary relativistic models of magnetic jets has to rely on simplifying assumptions such as self-similarity (Contopoulos 1994, 1995), some other prescription of the field structure (Li 1993; Beskin 1997) or the restriction to asymptotic regimes (Chiueh et al. 1991; Appl \& Camenzind 1993; Nitta 1994, 1995). For highly magnetized jets the forcefree limit applies. This allows for a truly two-dimensional calculation of the magnetic field structure (Fendt et al. 1995; Fendt 1997a). Relativistic magnetohydrodynamics implies that poloidal electric fields, which are not present in Newtonian MHD, cannot be neglected anymore.

From the observations we know that extragalactic jets as well as Galactic superluminal jets and protostellar jets are collimated almost to a cylindrical shape (Zensus et al. 1995; Ray et al. 1996; Mirabel \& Rodriguez 1995). Theoretically, it has been shown that current carrying relativistic jets must collimate to a cylinder (Chiueh et al. 1991). For the asymptotic limit of a cylindrically collimated magnetic relativistic jet, Appl \& Camenzind (1993) found a non-linear analytical solution for the trans-field force-balance in the case of a constant iso-rotation parameter. These results were further generalized for jets with differential rotation (Fendt 1997b). Such an asymptotic field distribution is especially interesting for jets emerging from (differentially rotating) accretion disks.

In previous papers, we applied the asymptotic jet model of Appl \& Camenzind (1993) as a boundary condition for the calculation of global, two-dimensional, forcefree jet magnetospheres for rapidly rotating stars (Fendt et al. 1995) or rapidly rotating black holes (Fendt 1997a). In this paper, we continue our work on $2 \mathrm{D}$ force-free jet magnetospheres applying an asymptotic jet with differential rotation of the iso-rotation parameter $\Omega_{\mathrm{F}}$ as boundary condition for the global jet structure. Such an approach allows for a connection between the differentially rotating jet source - the accretion disk - and the asymptotic collimated jet. Since jet motion seems intrinsically connected to the accretion disk, differential rotation of the field lines should be a natural ingredient for any magnetic jet structure. As a principal problem for differentially rotating relativistic jet magnetospheres, position and shape of the singular light surface are not known a priori, but have to be calculated in an iterative way together with the magnetic flux distribution.

In Sect. 2 we recall some basic equations of the theory of relativistic magnetospheres and discuss several difficulties with the solution of the Grad-Shafranov (hereafter GS) equation. After some comments on the numerical approach in Sect. 3, we discuss our results in Sect. 4. A summary is given in Sect. 5 .

\section{Structure of magnetic jets}

Throughout the paper we apply the following basic assumptions: axisymmetry, stationarity and ideal MHD. We use cylindrical coordinates $(R, \phi, Z)$ or $(x, \phi, z)$ if normalized. The term "asymptotic" always denotes the limit of $Z \gg R$ unless explicitly stated otherwise. We consider jets with a finite radius, $R<\infty$ for $Z \rightarrow \infty$.

\subsection{The force-free, cross-field force-balance}

With the assumption of axisymmetry, a magnetic flux function can be defined

$$
\Psi=\frac{1}{2 \pi} \int \boldsymbol{B}_{\mathrm{P}} \cdot \mathrm{d} \boldsymbol{A}, \quad R \boldsymbol{B}_{\mathrm{P}}=\nabla \Psi \wedge \boldsymbol{e}_{\phi}
$$

measuring the magnetic flux through a surface element with radius $R$ and, in a similar way, the poloidal current through the same area

$I=\int \boldsymbol{j}_{\mathrm{P}} \cdot \mathrm{d} \boldsymbol{A}=-\frac{c}{2} R B_{\phi}$

which, in the force-free case, flows parallel to the flux surfaces, $I=I(\Psi)$.

The structure of the magnetic flux surfaces is determined by the toroidal component of Ampère's law, $\nabla \times \boldsymbol{B}_{\mathrm{P}}=4 \pi \boldsymbol{j}_{\phi} / c$, where the toroidal electric current density has to be calculated from the equation of motion projected perpendicular to the flux surfaces (Camenzind 1987; Fendt et al. 1995). For strong magnetic fields, inertial forces of the matter can be neglected. This is the force-free limit and the equation of motion reduces to $0=c \rho_{\mathrm{c}} \boldsymbol{E}+\boldsymbol{j} \times \boldsymbol{B}$ with the charge density $\rho_{\mathrm{c}}$.

Combining Ampère's law and the force-free equation of motion the cross-field force-balance can be written as the modified relativistic GS equation,

$$
\begin{aligned}
R \nabla \cdot\left(\frac{1-\left(R \Omega_{\mathrm{F}}(\Psi) / c\right)^{2}}{R^{2}} \nabla \Psi\right)= & -\frac{4}{c^{2}} \frac{1}{R} \frac{1}{2}\left(I^{2}(\Psi)\right)^{\prime} \\
& -R|\nabla \Psi|^{2} \frac{1}{2}\left(\Omega_{\mathrm{F}}^{2}(\Psi)\right)^{\prime}
\end{aligned}
$$

where the primes denote the derivative $\frac{\mathrm{d}}{\mathrm{d} \Psi}$ (see Camenzind 1987; Okamoto 1992).

$\Omega_{\mathrm{F}}$ is conserved along the flux surfaces, $\Omega_{\mathrm{F}}=\Omega_{\mathrm{F}}(\Psi)$. We will call it the iso-rotation parameter, defined by Ferraro's law of iso-rotation. It can be understood as the angular velocity of the plasma, reduced by the slide along the field lines. Sometimes, it is called the angular velocity of the field lines. Both, the current distribution $I(\Psi)$ and the rotation law $\Omega_{\mathrm{F}}(\Psi)$ determine the source term for the GS equation and govern the structure of the magnetosphere. We apply the following normalization,

$$
\begin{aligned}
R, Z & \Leftrightarrow x R_{0}, z R_{0}, \\
\Omega_{\mathrm{F}} & \Leftrightarrow \Omega_{\mathrm{F}}\left(c / R_{0}\right), \\
\Psi & \Leftrightarrow \Psi \Psi_{\max }, \\
I & \Leftrightarrow I I_{\max } .
\end{aligned}
$$


As the length scale for the GS Eq. (3) the asymptotic radius $R_{0}$ of the light surface is selected (see below). In order to allow for a direct comparison to rigidly rotating magnetospheres, the normalization was chosen such that $\Omega_{\mathrm{F}}=1$ at $x=1$. With the chosen normalization, Eq. (3) can be written dimensionless,

$$
\begin{aligned}
x \nabla \cdot\left(\frac{1-x^{2} \Omega_{\mathrm{F}}^{2}(\Psi)}{x^{2}} \nabla \Psi\right)= & -\frac{1}{x} \frac{g}{2}\left(I^{2}(\Psi)\right)^{\prime} \\
& -x|\nabla \Psi|^{2} \frac{1}{2}\left(\Omega_{\mathrm{F}}^{2}(\Psi)\right)^{\prime} .
\end{aligned}
$$

$g$ is a coupling constant describing the strength of the current term in the GS equation,

$g=\frac{4 I_{\max }^{2} R_{0}^{2}}{c^{2} \Psi_{\max }^{2}}=4\left(\frac{I_{\max }}{10^{18} \mathrm{~A}}\right)^{2}\left(\frac{R_{0}}{10^{16} \mathrm{~cm}}\right)^{2}\left(\frac{\Psi_{\max }}{10^{33} \mathrm{Gcm}^{2}}\right)^{-2}$

where the parameters are chosen for extragalactic jets. In this paper, $g$ is in accordance with the definition in Fendt et al. (1995) and differs from the definition in Appl $\&$ Camenzind (1993) by a factor of two, $g_{\text {Fendt }}=2 g_{\mathrm{AC}}{ }^{1}$. Interestingly, a coupling constant, defined in a similar way also for the differential rotation term, would be equal to unity. The GS equation is numerically solved applying the method of finite elements (see Appendix).

Along the light surface, where $D \equiv 1-x^{2} \Omega_{\mathrm{F}}^{2}(\Psi)=0$, the GS equation reduces to the regularity condition,

$\nabla \Psi \cdot \nabla D=-g \frac{1}{2}\left(I(\Psi)^{2}\right)^{\prime}-\frac{1}{2}|\nabla \Psi|^{2}\left(\ln \left(\Omega_{\mathrm{F}}(\Psi)^{2}\right)\right)^{\prime}$,

which is equivalent to a Neumann boundary condition. However, for differentially rotating magnetospheres with $\Omega_{\mathrm{F}}=\Omega_{\mathrm{F}}(\Psi)$ the shape of this surface is not known a priori and has to be calculated in an iterative way together with the two-dimensional solution of the GS equation. For constant $\Omega_{\mathrm{F}}$ the light surface is of cylindrical shape. As we have shown in a previous publication (Fendt et al. 1995), our finite element code satisfies the regularity condition automatically, since the surface integral along the light surface vanishes.

\subsection{Discussion of the force-free assumption}

It is clear that relativistic jets must be highly magnetized. Only a high plasma magnetization gives jet velocities close to the speed of light (Fendt \& Camenzind 1996). Therefore, for the calculation of field structure the force-free limit seems to be reasonable. However, one may question the assumption of a force-free asymptotic jet. In a fully self-consistent picture of magnetic jet formation, the asymptotic jet is located beyond the collimating, non force-free wind region and beyond the fast magnetosonic

\footnotetext{
${ }^{1}$ Due to the fact that the jet radius (where $\Psi=1$ ) is not known before the asymptotic GS equation has been solved (Fendt 1997b), the normalization with $g$ leads to a current distribution $I(\Psi)$ which is not normalized to unity. This difference in normalization is "hidden" in the coupling constant $g$, which could, in principal, be re-scaled appropriately.
}

surface. The asymptotic jet parameters are determined by the wind motion. Thus, poloidal current and iso-rotation parameter of the field are not functions free of choice. The force-free region of a jet is located close to its origin, where the speed is low. Beyond the Alfvén surface plasma kinetic energy dominates the magnetic energy, which is just the contrary to force-freeness.

For small plasma density, the Alfvén surface of the wind flow approaches the light surface. In this case the fast magnetosonic surface moves to infinity for a conical flow. Okamoto (1999) argues that a force-free field distribution extending to infinity in both $x$ and $z$ direction will asymptotically be of conical shape, i.e. un-collimated. However, his approach differs from ours in the sense that he assumes that all field lines will cross the light cylinder. Such an assumption per se prohibits any collimation. On the other hand, perfect jet collimation is an observational fact. Astrophysical jets (of very different energy scales) appear collimated to cylinders of finite radius.

In general, the non force-free relativistic GS equation shows three inertial contributions,

$$
\begin{aligned}
0= & -\tilde{\kappa}\left(1-M^{2}-x^{2} \Omega_{\mathrm{F}}^{2}\right)+\left(1-x^{2} \Omega_{\mathrm{F}}^{2}\right) \frac{\nabla_{\perp} B_{\mathrm{P}}^{2}}{8 \pi}+\frac{\nabla_{\perp} B_{\phi}^{2}}{8 \pi} \\
& +\nabla_{\perp} P+\left(\frac{B_{\phi}^{2}}{4 \pi}-\rho u_{\phi}^{2}\right) \frac{\nabla_{\perp} x}{x}-\frac{B_{\mathrm{P}}^{2} \Omega_{\mathrm{F}}}{4 \pi} \nabla_{\perp}\left(x^{2} \Omega_{\mathrm{F}}\right),
\end{aligned}
$$

where $\nabla_{\perp}$ indicates the gradient perpendicular to $\Psi, \tilde{\kappa} \equiv$ $\kappa B_{\mathrm{P}}^{2} / 4 \pi=\boldsymbol{n} \cdot\left(\boldsymbol{B}_{\mathrm{P}} \cdot \nabla\right) \boldsymbol{B}_{\mathrm{P}} / 4 \pi$ the poloidal field curvature, $\rho$ the mass density, $u_{\phi}$ the toroidal velocity, $P$ the gas pressure and $M$ the Alfvén Mach number (Chiueh et al. 1991). One can show that in the asymptotic, cylindrical jet the contribution of inertial terms in the force-balance across the field is weak. The contribution of gas pressure is usually negligible in astrophysical jets. Also, the centrifugal force does not play a role for radii larger than the Alfvén radius, since outside the Alfvén surface (where $M^{2}=1-x^{2} \Omega_{\mathrm{F}}^{2}$ ) the plasma moves with constant angular momentum. The curvature term vanishes in cylindrical geometry. Therefore, since for cylindrical jets the contribution from inertial terms is weak, the configuration is comparable to the force-free case. The source term of the GS equation may be reduced to a form similar to the common force-free limit. We suggest the phrase "quasi force-free" for such a configuration because the GS equation looks force-free even if the physical system is not magnetically dominated.

In the force-free limit of a highly magnetized plasma the previous arguments also apply. However, in difference to the asymptotic regime considered above, the low plasma density implies that inertial terms are weak over the whole two-dimensional jet region. The centrifugal term $\rho u_{\phi}$ is weak even if the Alfvén surface now comes close to the light surface. Numerical calculations of the plasma motion along the field have shown that, for a high magnetization, the Alfvén Mach number $M$ grows almost linearly with radius but remains relatively low (Fendt \& Camenzind 1996). Thus, the inertial curvature term should not play a 
dominant role. Contopoulos \& Lovelace (1994) find from self-similar solutions that centrifugal forces are dominated by magnetic forces leading to a re-collimation of the outflow.

In summary, our discussion of the inertial terms in the force-balance equation has shown that these terms are generally weak in the case of a high magnetization. We therefore think that for the calculation of the magnetic field structure in relativistic jets the force-free assumption is acceptable. The main motivation of the force-free assumption is clearly the reason of simplification. There is yet no other way to calculate a truly two-dimensional field distribution for relativistic jets. Naturally, with a forcefree solution, nothing can be said about the flow acceleration itself.

\subsection{Location of the asymptotic light cylinder}

The radius of the asymptotic light cylinder $R_{0}$ is the natural length-scale for the GS solution. A scaling of the GS solution in terms of the properties of the central object (e.g. its mass) relies on the proper connection between the asymptotic jet and the accretion disk. This is feasible only if differential rotation $\Omega_{\mathrm{F}}(\Psi)$ is included in the treatment (see Sect. 3).

In the following we consider the location of the light surface and its relation to the relativistic character of the magnetosphere from a general point of view. The light cylinder of a flux surface $\Psi$ is defined as a cylinder with radius $R=R_{\mathrm{L}}(\Psi) \equiv c / \Omega_{\mathrm{F}}(\Psi)$. At this position the GS equation becomes singular. However, this light cylinder is only important for the field line if the field line actually intersects it as for $\Psi_{\text {out }}$ in Fig. 1. Only then, relativistic effects become dominant. For example, the poloidal electric field scales with the radius in units of the light cylinder radius, $E_{\mathrm{P}}=\left(R / R_{\mathrm{L}}\right) B_{\mathrm{P}}$. On the other hand, in the case of $\Psi_{\text {in }}$ in Fig. 1, the asymptotic radius of the flux surface is smaller than its light cylinder radius $R_{\mathrm{L}}\left(\Psi_{\text {in }}\right)$ (located between $\Psi_{\text {in }}$ and $\left.\Psi_{0}\right)$, therefore relativistic effects are small. For jet solutions with rigid rotation $\Omega_{\mathrm{F}}$ all flux surfaces have the same light cylinder radius. Thus, the singular light surface of the magnetosphere is a cylinder. For jet solutions with differential rotation $\Omega_{\mathrm{F}}$ the flux surfaces have different light cylinder radii. The singular surface of the magnetosphere is not a cylinder anymore.

It is now interesting to note that the case of differential rotation $\Omega_{\mathrm{F}}(\Psi)$ allows for a hypothetical field distribution where (i) the light radius of most of the flux surfaces is located within the jet radius, but where also (ii) the asymptotic radius of the flux surfaces is always smaller than their light radius. Such a field distribution would not have a singular light surface and could be considered as "non relativistic", even if the hypothetical light radii of many field lines are inside the jet radius. Such a situation is not possible if the magnetosphere is constrained by a constant rotation $\Omega_{\mathrm{F}}$. This underlines the importance of the treatment of differential rotation for jets from accretion disks.

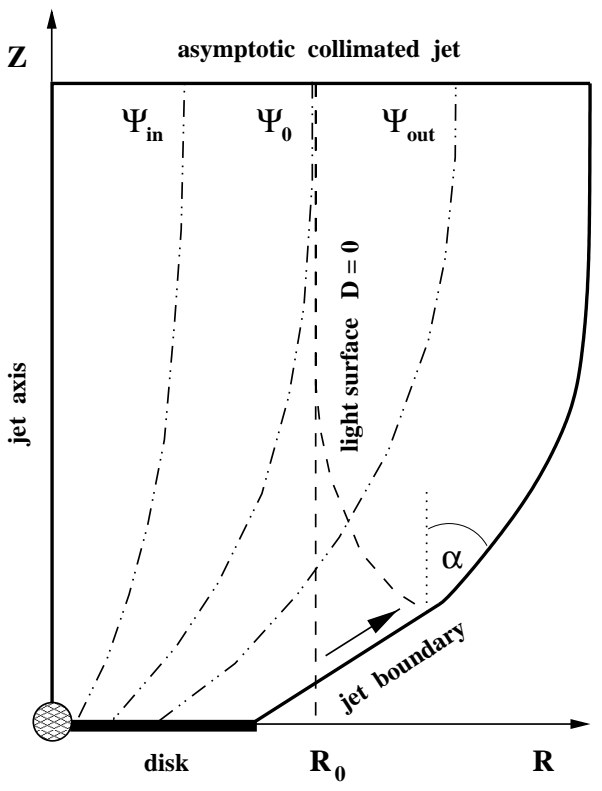

Fig. 1. Sketch of the jet model. Axisymmetric jet magnetic flux surfaces $\Psi$ projected into the meridional plane. The central object, located within the inner boundary (solid disk), is surrounded by an accretion disk. Helical magnetic field lines (laying on the flux surfaces) are anchored in the differentially rotating disk at the foot points $R_{\mathrm{D}}(\Psi)$. The jet boundary is defined by the flux surface $\Psi=1$. The upper boundary condition is a cylindrically collimated jet solution (Fendt 1997b). The arrow indicates the numerical deformation of the initially vertical boundary of the inner solution (at $x=1$ ) into the curved light surface. The flux surfaces $\Psi_{\text {in }}\left(\Psi_{\text {out }}\right)$ have an asymptotic radius smaller (larger) than the asymptotic light cylinder $R_{0}$, which is the asymptotic branch of the light surface $R_{\mathrm{L}}(\Psi)$ for large $z$. The flux surface $\Psi_{0}$ coincides with the light surface asymptotically. The jet half opening angle is $\alpha$ (see Sect. 2.4, Fig. 2)

A relativistic description for the jet magnetosphere is always required if the jet contains a flux surface for which the light radius is smaller than the asymptotic radius.

\subsection{The regularity condition and the jet opening angle}

The regularity condition (5) is the natural boundary condition along the light surface. Although it is impossible to solve Eq. (5) explicitly, a general relation concerning the jet opening angle can be derived. First, we rewrite Eq. (5) as

$B_{z}=\frac{1}{4} g\left(I^{2}\right)^{\prime}-\frac{1}{4} B_{\mathrm{P}}^{2}\left(\frac{1}{\Omega_{\mathrm{F}}^{2}}\right)^{\prime}$,

where $\Omega_{\mathrm{F}}(\Psi)=1 / x_{\mathrm{L}}(\Psi) \equiv R_{0} / R_{\mathrm{L}}(\Psi)$ has been applied. From Eq. (6) it follows for the radial field component $B_{x}^{2}=$ $-g\left(I^{2}(\Psi)\right)^{\prime} /\left(1 / \Omega_{\mathrm{F}}^{2}(\Psi)\right)^{\prime}$, if $\Psi$ intersects the light surface with vanishing $B_{z}$. On the other hand, considering a field line perpendicular to the light surface, $\nabla \Psi \perp \nabla D$, this provides a condition for the axial field component,

$B_{z}=\frac{g}{2}\left(I^{2}\right)^{\prime}=\frac{B_{\mathrm{P}}^{2}}{2}\left(\frac{1}{\Omega_{\mathrm{F}}^{2}}\right)^{\prime}$. 

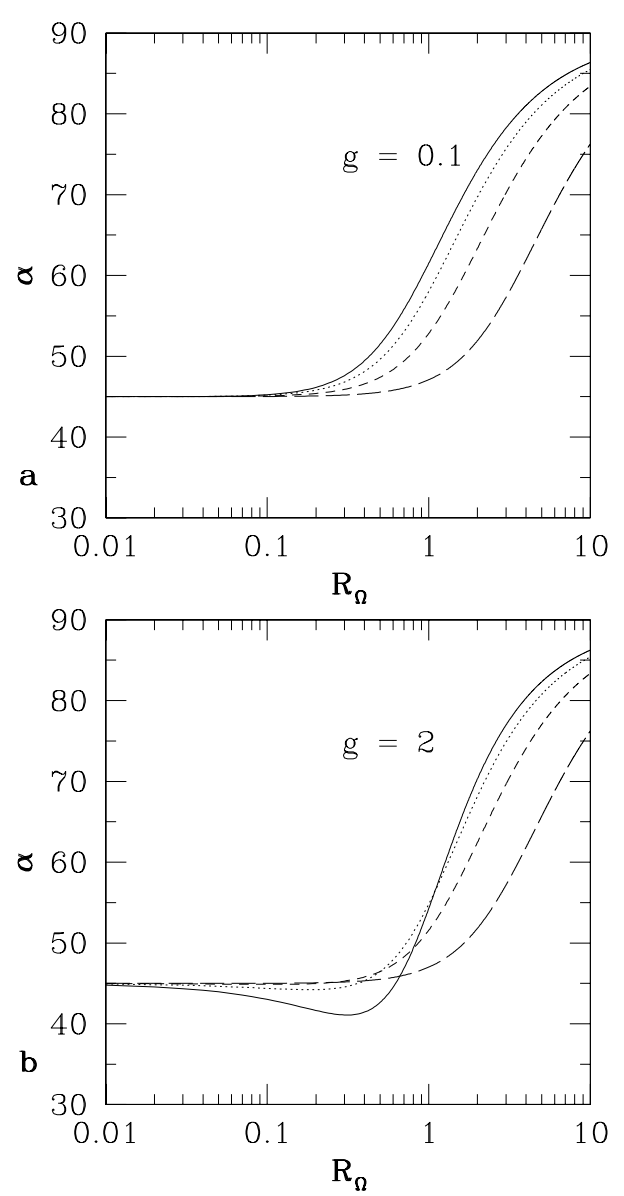

Fig. 2. Jet half opening angle $\alpha(\Psi=1)$ for the analytical asymptotic jet solution and along the asymptotic branch of the light surface in $x$-direction (see Eq. (8)). Coupling constant $g=0.1 \mathbf{a}$ ) and $g=2 \mathbf{b}$ ). Asymptotic magnetic flux distribution parameter (Eq. (9)) $b=0.5$ (solid), $b=1$ (dotted), $b=2$ (short-dashed), $b=5$ (long-dashed)

Interestingly, this is either only a function of the current distribution $I(\Psi)$ or depends only from the specification of the rotation law $\Omega_{\mathrm{F}}(\Psi)$. Further, in this case it is always $B_{z}>0$, since $\left(1 / \Omega_{\mathrm{F}}^{2}\right)^{\prime}=\left(x_{\mathrm{L}}^{2}\right)^{\prime}>0$. In particular, for the asymptotic $(z \rightarrow \infty)$ part of the magnetosphere, this implies that only collimating field lines can cross the light surface.

Now we consider the asymptotic branches of the light surface. For the asymptotic branch in $z$-direction it holds $(\nabla D)_{x} \gg(\nabla D)_{z} \simeq 0$. Further, it is $B_{x}\left(\ln \Omega_{\mathrm{F}}^{2}\right)^{\prime}=0$, implying either a collimated field structure, $B_{x} \equiv 0$ or rigid rotation, $\left(\Omega_{\mathrm{F}}(\Psi)\right)^{\prime} \equiv 0$. From this we conclude that in the asymptotic regime of a cylindrical light surface, also the flux surfaces along this light cylinder must be of cylindrical shape. Collimation must occur in the non-asymptotic region of the jet.

If we now assume that there exists an asymptotic part of the light surface in $x$-direction (where $x \gg z$ ) and that $(\nabla D)_{z} \gg(\nabla D)_{x} \simeq 0$, we derive an equation for the half jet opening angle,

$\alpha(\Psi)=\tan ^{-1}\left(\sqrt{1+\frac{1}{4} g \frac{\left(I^{2}(\Psi)\right)^{\prime}\left(\Omega_{\mathrm{F}}^{2}(\Psi)\right)^{\prime}}{\Omega_{\mathrm{F}}^{4}(\Psi)}}\right)$, for the flux surfaces in this region. As a general example we apply the analytical solution obtained for the asymptotic jet (Fendt 1997b),

$$
\begin{aligned}
\Psi(x) & \equiv \frac{1}{b} \ln \left(1+\left(\frac{x}{a}\right)^{2}\right), \quad b \equiv \ln \left(1+\left(\frac{x_{\mathrm{jet}}}{a}\right)^{2}\right), \\
\Omega_{\mathrm{F}}^{2}(\Psi) & =\frac{g b^{2}}{4}\left(\frac{I^{2}(\Psi)}{\left(1-\mathrm{e}^{-b \Psi}\right)^{2}}-\frac{1}{\left(1-\mathrm{e}^{-b}\right)^{2}}\right)+\Omega_{\mathrm{F}}^{2}(1)
\end{aligned}
$$

for Eq. (9). Here, $b$ is a measure for the ratio of jet radius to jet core radius $a$. Finally, we obtain the half opening angle for the outermost flux surface $\Psi=1$,

$\alpha=\tan ^{-1}\left(\sqrt{1+R_{\Omega}\left(4 R_{\Omega}\left(\frac{\mathrm{e}^{b}-1}{b \mathrm{e}^{b}}\right)^{2}+\frac{g / 2}{\mathrm{e}^{b}-1}\right)}\right)$,

where $R_{\Omega}$ is defined as $\left(\Omega_{\mathrm{F}}(1)\right)^{\prime} / \Omega_{\mathrm{F}}(1)$. Note, that Eq. (10) is only valid in the limit of $(\nabla D)_{x} \simeq 0$. Figure 2 shows the variation of the opening angle $\alpha$ with the parameters $a$ and $b$ for two choices of the strength of the poloidal electric current. In general, jets with a strong differential rotation $\Omega_{\mathrm{F}}(\Psi)$ (i.e. large $R_{\Omega}$ ) have a larger opening angle. Also, jets with a large ratio of jet to core radius have a smaller opening angle. Therefore, jets originating in a small part of the accretion disk, equivalent to small value of $R_{\Omega}$, will be collimated to a smaller opening angle. It is interesting to note that, in the case of a rigid rotation, the limiting half opening angle is $45^{\circ}$, independently of $g$ and $b$.

\section{The jet-disk connection, providing the true length scale of the GS solution}

The natural length scale of the relativistic GS Eq. (3) is the asymptotic light cylinder $R_{0}$ (see Sect.2.3). Its size is related to the iso-rotation parameter $\Omega_{\mathrm{F}}(\Psi)$, which itself is connected to the angular rotation of the foot points of the field lines. Concerning the GS equation, the size of $R_{0}$ follows purely from electro-magnetic quantities, if the coupling constant $g$ is chosen. The GS solution can be scaled to any central object from stars to galactic nuclei as long as the interrelation of the parameters $\Psi_{\max }, I_{\max }$ and $R_{0}$ provides the same $g$. So far, no connection has been made to the type of central object. Here, we treat the question where the asymptotic light cylinder is located in physical units.

In the case of rigid rotation, the light cylinder radius is usually estimated by choosing a distinct radial distance from the central object and defining $\Omega_{\mathrm{F}}$ under the assumption that the jet magnetosphere is anchored in that point. If the central object is a black hole, the marginally stable orbit implies an upper limit for $\Omega_{\mathrm{F}}$. For jets in AGN this estimate leads to the common conclusion that the light cylinder radius is at about $10 R_{\mathrm{S}}$ and the typical jet radius at about $100 R_{\mathrm{L}}$. Clearly, such arguments relies on the assumption that the field line emerging at this very special radius defining $\Omega_{\mathrm{F}}$ also extends to the light cylinder radius $R_{\mathrm{L}}$ (see Sect. 2.3). 
This picture changes, if differential rotation $\Omega_{\mathrm{F}}(\Psi)$ is considered. Different flux surfaces anchor at different foot point radii and have different light radii (Sect. 2.3). Assuming a Keplerian rotation, the light surface radius $R_{\mathrm{L}}(\Psi)$ is located at

$R_{\mathrm{L}}(\Psi)=410^{15} \mathrm{~cm}\left(\frac{R_{\mathrm{D}}(\Psi)}{R_{\mathrm{S}}}\right)^{3 / 2}\left(\frac{M}{10^{10} M_{\odot}}\right)$,

where $R_{\mathrm{S}}$ is the Schwarzschild radius of a point mass and $R_{\mathrm{D}}(\Psi)$ the foot point of the flux surface $\Psi$ on a Keplerian disk. A more general equation is

$\frac{R_{\mathrm{L}}(\Psi)}{R_{\mathrm{S}}}=\sqrt{2}\left(\frac{R_{\mathrm{D}}(\Psi)}{R_{\mathrm{S}}}\right)^{3 / 2}$.

$R_{\mathrm{D}}(\Psi)$ is determined from the magnetic flux distribution along the disk and is defined by a certain disk model. Figure 3 shows the location of the light radius $R_{\mathrm{L}}$ for a field line anchored at a foot point radius $R_{\mathrm{D}}$ in a Keplerian disk around a central object of mass $M$ (see Eq. (11)). Note that the unit of the field line footpoint radius in Eq. (12) and Fig. 3 is the Schwarzschild radius. Therefore, Fig. 3 is appropriate only for relativistic jets. The footpoint radii for protostellar jets are several stellar radii, corresponding to about $10^{6}$ Schwarzschild radii (which would be located far above the box in Fig. 3).

So far, nothing can be said about the location of the asymptotic radius of the field lines in general. The essential question is where the asymptotic radius of a flux surface is located in respect to its light cylinder. This question can only be answered by a detailed model considering the two-dimensional field distribution including differential rotation $\Omega_{\mathrm{F}}(\Psi)$. Only in such a model, the flux distribution of the asymptotic jet can be connected to the flux distribution of the "star"-disk system. Certainly, both boundary conditions - asymptotic jet and accretion disk rely on model assumptions. However, in a self-consistent model these boundary conditions have to follow certain constraints (see Sects. 4.1, 4.3).

\section{The two-dimensional jet solution}

\subsection{Disk and jet boundary condition}

Three important boundary conditions determine the two-dimensional flux distribution. The first boundary condition is in the asymptotic region. Here we assume a cylindrically collimated jet. We apply the magnetic flux distribution derived by Fendt (1997b), where the rigidly rotating jet model of Appl \& Camenzind (1993) is extended for differential rotation $\Omega_{\mathrm{F}}(\Psi)$. In particular, our asymptotic jet shows the typical jet core-envelope structure of magnetic flux and electric current, i.e. a configuration where most of the magnetic flux and poloidal electric current is concentrated within a "core" radius. The asymptotic model provides not only the asymptotic magnetic flux boundary condition but also the $\Omega_{\mathrm{F}}(\Psi)$ and $I(\Psi)$ distribution for the whole two-dimensional jet magnetosphere. In the model of Fendt (1997b) these functions

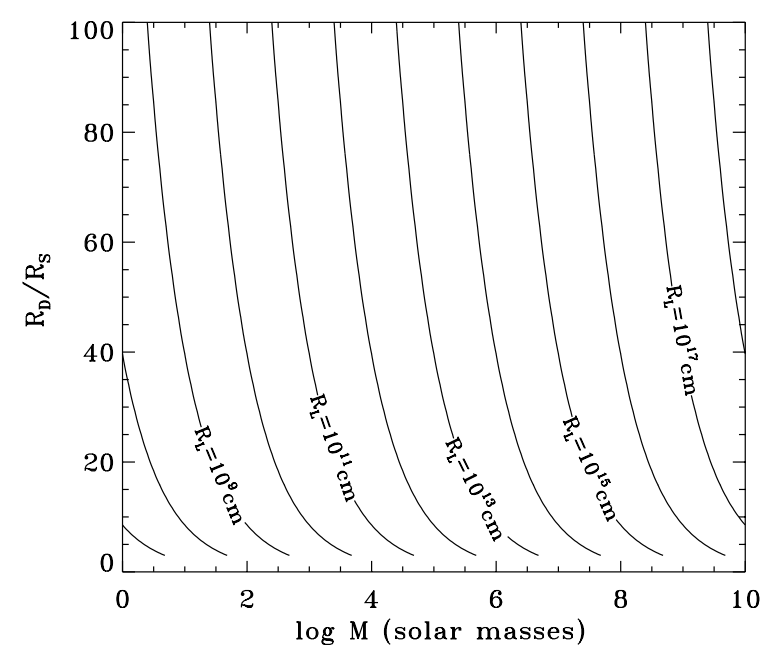

Fig. 3. Location of the light cylinder radius of a flux surface $R_{\mathrm{L}}(\Psi)$, anchored at a certain foot point radius $R_{\mathrm{D}}(\Psi)$ in units of the Schwarzschild radius $R_{\mathrm{S}}$ in a Keplerian disk around a point mass $M$. Note that for non collapsed stellar objects the footpoint radii of the jet field lines are located at about $10^{6} R_{\mathrm{S}}$

follow from the solution of the one-dimensional (asymptotic) GS equation across the cylindrical jet and the prescription of $I(x)=(x / a)^{2} /\left(1+(x / a)^{2}\right)$ together with $\Omega_{\mathrm{F}}^{2}(x)=\mathrm{e}^{h-h x}$, where $a$ is the core radius of the electric current distribution and $h$ governs the steepness of the $\Omega_{\mathrm{F}}$-profile ${ }^{2}$.

The second boundary condition is the magnetic flux distribution along the disk. This distribution is in general not known as a solution of the full MHD disk equations. Typical models rely on various simplifying assumptions, as e.g. stationarity, the distribution of magnetic resistivity or the disk turbulence governing a dynamo process. We apply an analytic flux distribution similar to the model of Khanna \& Camenzind (1992), who calculated the stationary accretion disk magnetic field structure around a super massive black hole. The typical behavior of the magnetic flux distribution is (i) a slight increase of magnetic flux along the innermost disk, (ii) a small or vanishing flux at the inner disk radius, (iii) a strong increase of magnetic flux at intermediate radius (the core radius) and (iv) a saturating behavior for large radii. Using the normalization introduced above, we choose the following disk boundary magnetic flux distribution,

$\Psi_{\mathrm{disk}}(x)=\frac{1}{\tilde{b}} \ln \left(1+\left(\frac{x-x_{\mathrm{in}}}{\tilde{a}}\right)^{2}\right)$

with $\left.\tilde{b}=\ln \left(1+\left(x_{\mathrm{disk}}-x_{\mathrm{in}}\right)^{2} / \tilde{a}^{2}\right)\right)$ (see Fig. 4). The parameters are: the core radius $\tilde{a}$, the disk outer radius, $x_{\text {disk }}$ and the disk inner radius, $x_{\text {in }}$. For simplicity, we choose $x_{\text {in }} \simeq 0$ without loss of generality. Such a choice will definitely not influence the global jet solution which is normalized to the asymptotic light cylinder radius.

\footnotetext{
${ }^{2}$ For figures of these functions and the related $\Psi(x), \Omega_{\mathrm{F}}(\Psi)$ and $I(\Psi)$ distribution, we refer to Fendt (1997).
} 


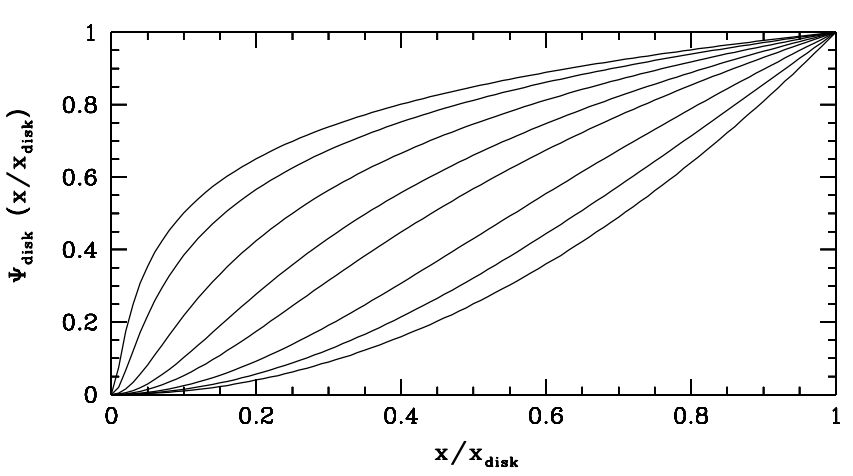

Fig. 4. Magnetic flux distribution along the disk $\Psi_{\text {disk }}(x)$ as defined in Eq. (13). Parameters: $x_{\text {in }}=0, x_{\text {disk }} / \tilde{a}=100$, $40,15,7,4,2,1,0.01$

The third boundary condition is the jet boundary $x_{\text {jet }}(z)$. Along this boundary the flux distribution is $\Psi=1$ by definition. However, the shape of this boundary is not known a priori. It must be determined by the regularity of the solution across the light surface (see also Fendt et al. 1995). A slightly different shape may give the same solution. However, the main features of the solution as opening angle or locus of the collimation are fixed by the internal equilibrium. Therefore, the regularity condition governs the shape of the jet boundary. For a given $I(\Psi), \Omega_{\mathrm{F}}(\Psi)$, disk and jet boundary condition, the jet radius $x_{\text {jet }}(z)$ is uniquely determined.

The inner spherical grid boundary with radius $x_{\star}$ close to the origin, indicates the regime of the central source, possibly enclosing a collapsed object. Neutron stars or magnetic white dwarfs may carry their own magnetic field, a black hole can only be threaded by the disk magnetic field. In any case, the magnetic flux distribution is a combination of "central" magnetic flux and disk magnetic flux $\Psi=\Psi_{\star}+\Psi_{\text {disk }}$. For simplicity we assume that the magnetic flux increases monotonically from the axis to the disk edge and $\Psi\left(x_{\star}\right)=\Psi_{\star}\left(x_{\star}\right)$ and $\Psi_{\text {disk }}\left(x_{\star}\right)=0$.

\subsection{The two-dimensional collimating magnetic field structure}

Results of numerical solutions of the GS equation are presented in Fig. 5. Shown is the two-dimensional structure of the magnetic flux surfaces as projection of the helical field lines onto the meridional plane. In general, for a choice of the "free functions" $I(\Psi)$ and $\Omega_{\mathrm{F}}(\Psi)$, here taken from the asymptotic cylindrical jet solution, the field structure is determined by the boundary conditions and the regularity condition along the light surface.

We calculated two solutions with a different choice for the steepness parameter in the iso-rotation $\Omega_{\mathrm{F}}$. The first solution is for $h=0.2$ (Fig. 5, left). This is more close to the case of rigid rotation. Indeed the solution look rather similar to the solutions presented in Fendt et al. (1995). The second solution is for $h=0.5$ (Fig. 5, right). The steeper profile for the rotation law implies a smaller asymptotic jet radius (Fendt 1997b). This can be seen in comparison with the rigid rotation solutions (Fendt et al. 1995). However, a larger poloidal electric current can balance the effect of differential rotation. Therefore, the $h=0.2$ solution (with $g=2.5$ ) collimates to a smaller asymptotic jet radius than the $h=0.5$ solution (with $g=2.0)$. A $h=0.2$ solution with $g=2.0$ would have a jet radius of 2.4. The second solution with the steeper profile of the rotation law $\Omega_{\mathrm{F}}(\Psi)$ would better fit to a Keplerian disk rotation. A perfect match would require an even steeper $\Omega_{\mathrm{F}}(\Psi)$-profile (see below).

The mean half opening angle of the jet magnetospheres is about $60^{\circ}$. As discussed above, the shape of the outermost flux surface $(\Psi=1)$ is not prescribed but is a result of our calculation eventually determined by the regularity condition. After crossing the light surface the jets collimate to their asymptotic radius within a distance from the source of about $1-2 R_{0}$ along the jet axis. The opening angle of the second solution is smaller, however, the jet collimation is achieved only at a larger distance from the central source. In our examples, the "jet expansion rate", which we define as the ratio of the asymptotic jet radius to the foot point jet radius (the "disk radius"), is about 10. The true scaling of the jet magnetosphere in terms of the size of the central object can be determined by connecting the jet iso-rotation parameter $\Omega_{\mathrm{F}}(\Psi)$ to the disk rotation (see next section).

We note that, although in our computations the jet boundary $x_{\text {jet }}(z)$ is determined by the force-balance within the jet, and therefore subject to the regularity condition, with our results we do not prove the magnetohydrodynamic self-collimation process of the jet flow. Clearly, the calculated jet magnetosphere is self-collimated in the sense that its structure has been determined only by the internal properties. However, the actual collimation process of the jet flow from an un-collimated conical outflow into a cylinder could only be investigated by time-dependent simulations taking into account the interaction with the ambient medium.

On the other hand, we can assume that our jet solution is embedded in an ambient medium. If we further assume an equilibrium between the internal pressure (magnetically dominated) and external (gas) pressure along the jet boundary, we may derive the gas pressure distribution in the ambient medium, since we know the magnetic pressure distribution along the collimating jet radius. In this case, the jet solution may be considered as collimated by ambient pressure.

To our understanding one may claim a self-collimation only, if the jet flow collimates independently from external forces. Since in our treatment we do not consider the interrelation with the medium outside the jet, we cannot decide whether the flow is self-collimated or pressure collimated.

The field structure is governed by the choice of the functions $I(\Psi)$ and $\Omega_{\mathrm{F}}(\Psi)$, here taken from an asymptotic jet solution. In combination with the disk magnetic flux distribution (13) we can determine two parameters interesting for the jet-disk interaction. These are (i) the 

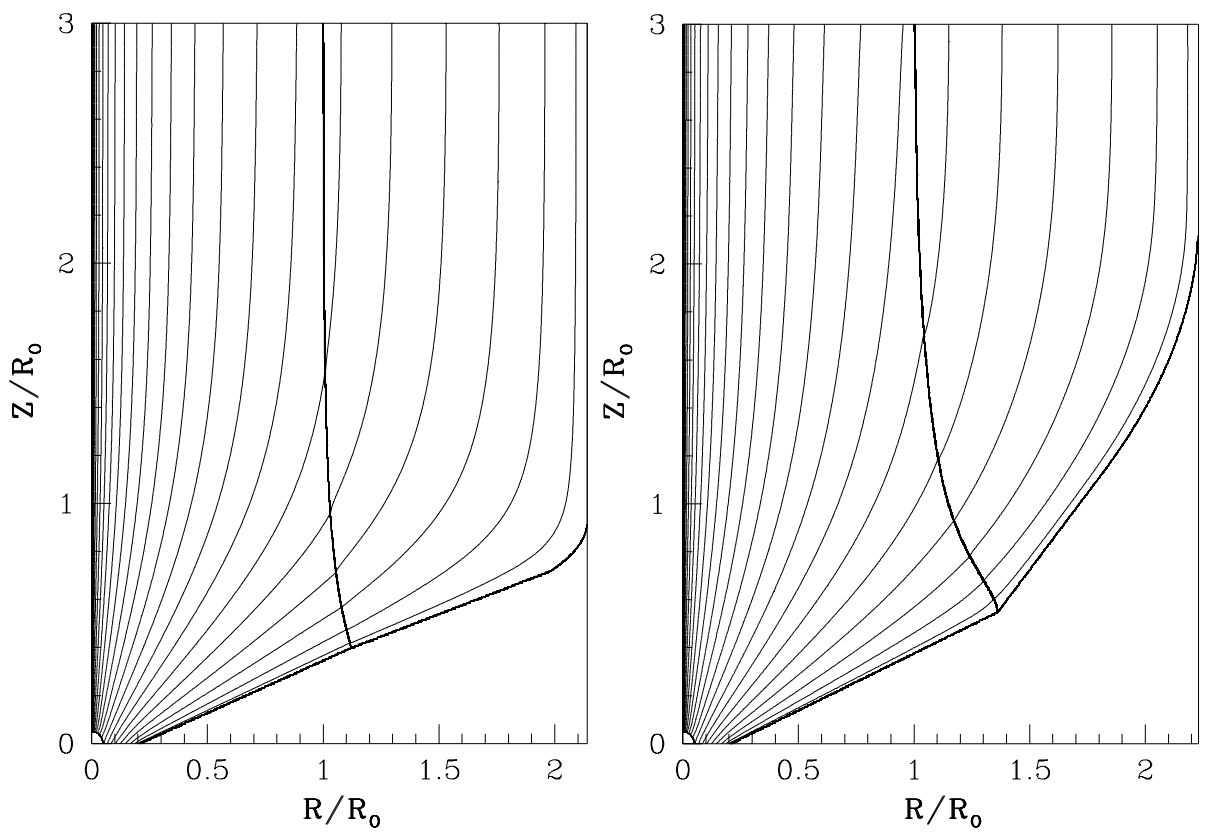

Fig. 5. Two-dimensional magnetic flux distribution $\Psi(x, z)$ for two different asymptotic rotation laws. Left: $h=0.2, g=2.5$ Right: $h=0.5, g=2.0$. Shown are iso-contours of the magnetic flux (equivalent to poloidal magnetic field lines) with contour levels $\Psi_{n}=10^{-(0.1 n)^{2}}, n=0, \ldots, 25$. Note that due to the choice of contour levels the iso-contour density does not mirror the field strength

magnetic angular momentum loss per unit time and unit radius from disk into the jet and (ii) the toroidal magnetic field distribution along the disk. With $I(\Psi)$ as the angular momentum flux per unit time per unit flux tube, the (normalized) angular momentum flux per unit time per unit radius is $\mathrm{d} \dot{J} / \mathrm{d} x=-x B_{z} I(x)$ along the disk. Figure 6 shows the behavior of both quantities for our jet model with the steeper profile of the rotation law, $h=0.5$. As we see, most of the magnetic angular momentum is lost in the outer parts of the disk. This may have interesting applications for accretion disk models taking into account a magnetized wind as a boundary condition. The total magnetic angular momentum loss is determined by the normalization, $\dot{J}=-\left(I_{\max } \Psi_{\max } / c\right) \int I(\Psi) \mathrm{d} \Psi$ or $\dot{J}=-\left(\sqrt{g} \Psi_{\max } / 2 R_{0}\right) \int I(\Psi) \mathrm{d} \Psi$. The magnetic toroidal field distribution along the disk has a maximum at about half the disk radius.

Clearly, these parameters are biased by the magnetic flux disk boundary condition (13) of our model. However, we believe that the main features are rather general and valid for any poloidal current and magnetic flux distribution with the typical core-envelope structure.

\subsection{Scaling relations of disk and jet}

As discussed above, the two-dimensional magnetic field distribution connecting the asymptotic jet region with the lower disk boundary allows for a direct scaling of the jet in terms of the size of the central object. This is simply based on the assumption that the foot points of the field lines are rotating with Keplerian speed, $\Omega_{\mathrm{F}}=\Omega_{\mathrm{K}}$ and to the fact that in ideal MHD the iso-rotation parameter $\Omega_{\mathrm{F}}$
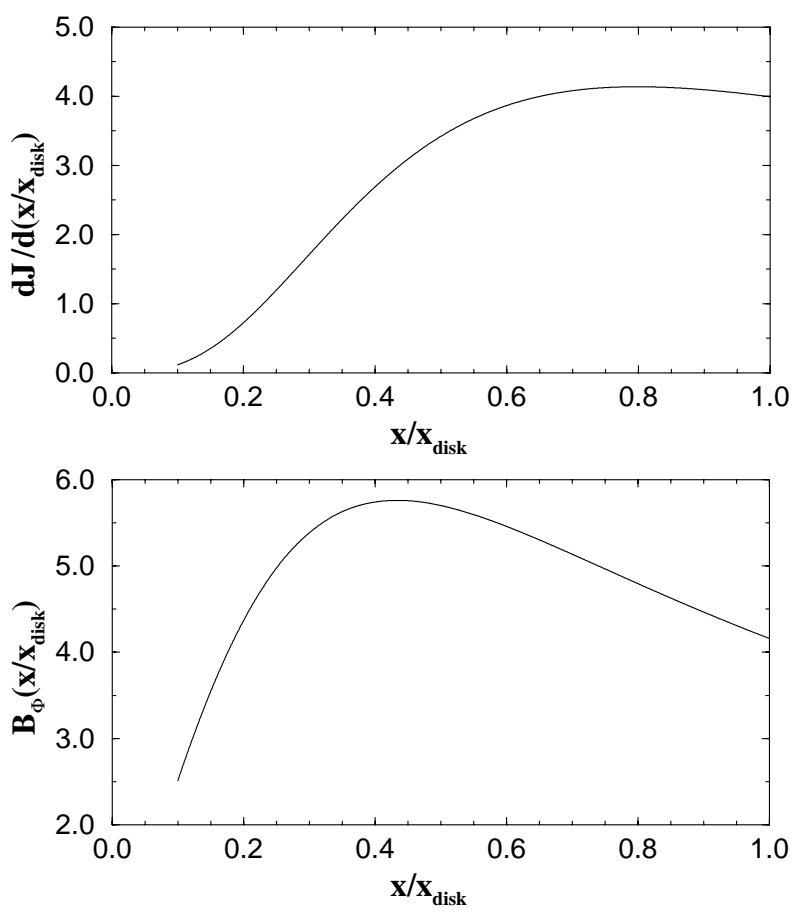

Fig. 6. Magnetic angular momentum loss per time unit per unit radius $\mathrm{d} \dot{J} / \mathrm{d} x$ at radius $x$ (above) and disk toroidal field distribution $B_{\phi}(x)$ (below) for the jet solution with $h=0.5$ shown in Fig. 5

is conserved along the field lines. It is therefore possible to construct a self-consistent model of the whole "star"disk-jet system with only a small set of free parameters. In the following we will motivate such a model. 
The first example demonstrates how the connection between the asymptotic jet and the disk, applied for our very special model assumption, provides a specific estimate for the asymptotic light cylinder $R_{0}$. Normalizing the Keplerian velocity $\Omega_{\mathrm{K}}$ in the same way as $\Omega_{\mathrm{F}}$ (Sect. 2.1), we obtain the expression

$R_{0}=\frac{G M}{c^{2} \Omega_{\mathrm{K}}^{2}} \frac{1}{x^{3}}=\frac{G M / c^{2}}{\Omega_{\mathrm{F}}^{2}(\Psi=1) x_{\mathrm{disk}}^{3}}=\frac{0.5 R_{\mathrm{S}}}{\Omega_{\mathrm{F}}^{2}(\Psi=1) x_{\mathrm{disk}}^{3}}$

Iso-rotation parameter $\Omega_{\mathrm{F}}(\Psi)$ and disk radius $x_{\text {disk }}$ are fixed by our model. Therefore, the asymptotic light cylinder is proportional to the mass of the central object. For $\Omega_{\mathrm{F}}^{2}(1)=0.54$ (which refers to the $h=0.5$ model) and $x_{\text {disk }}=0.2$ the asymptotic light cylinder is $R_{0}=116 R_{\mathrm{S}}$, which is about 2 times larger compared to the jet solution with a rigid rotation $\Omega_{\mathrm{F}} \equiv 1$ and will increase for larger values of $h$. With the choice of $g$, the value of $R_{0}$ constraints the maximum poloidal magnetic flux and electric current. Here, no assumption is made about the flux distribution along the disk.

In the second example we determine the disk magnetic flux distribution $\Psi(x)$ combining the asymptotic jet rotation law $\Omega_{\mathrm{F}}(\Psi)$ with a Keplerian disk rotation $\Omega_{\mathrm{K}}(x)$. From Eq. (14) follows that $\Omega_{\mathrm{F}}(\Psi) / \Omega_{\mathrm{F}}(1)=$ $\Omega_{\mathrm{K}}(x) / \Omega_{\mathrm{F}}(\Psi=1)=\left(x / x_{\text {disk }}\right)^{-3 / 2}$. In combination with the numerically derived $\Omega_{\mathrm{F}}(\Psi)$ this gives the $\Psi(x)$ along the disk (Fig. 7). The figure shows that the disk flux distribution derived from the asymptotic jet is distributed only over the outer part of the disk. This can be interpreted in two ways. First it may imply a relatively large inner disk radius and, hence, an asymptotic jet radius small in terms of radii of the central object. Secondly, it just underlines the fact that in our model the distribution of the asymptotic jet iso-rotation parameter is too flat in order to be truly connected to a disk magnetic flux with an extended radial distribution. For a model taking into account the disk Keplerian rotation in a fully self-consistent way, the magnetic flux distribution which has to be used as disk boundary condition for the GS solution is the one derived in Fig. 7.

On the other hand, the assumption of a Keplerian disk rotation together with a certain disk magnetic flux distribution provides an expression for the iso-rotation parameter $\Omega_{\mathrm{F}}(\Psi)=\Omega_{\mathrm{K}}(x(\Psi))=$ $\left(G M / R_{0} c^{2}\right)\left(\tilde{a}^{2}\left(\mathrm{e}^{\tilde{b} \Psi}-1\right)\right)^{-3 / 2}$. Here, the disk magnetic flux distribution (13) has been used. Eventually, one finds

$\frac{\Omega_{\mathrm{K}}(\Psi)}{\Omega_{\mathrm{F}}(1)}=\left(\frac{x_{\mathrm{disk}}}{\tilde{a}}\right)^{3 / 2}\left(\left(1+\left(\frac{x_{\mathrm{disk}}}{\tilde{a}}\right)^{2}\right)^{\Psi}-1\right)^{-3 / 4}$.

This function is definitely steeper compared to the $\Omega_{\mathrm{F}}(\Psi)$ distributions which have been derived in Fendt (1997b) and are used in the present paper. Here, we see the limitation of our ansatz. A steeper profile for rotation law is not yet possible to treat with our code due to the lack of numerical resolution. The non-linear character of the GS

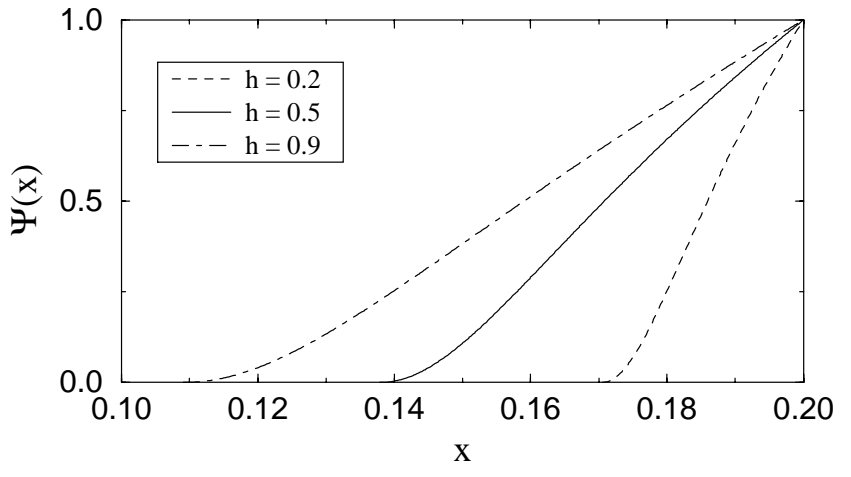

Fig. 7. Magnetic flux distribution $\Psi(x)$ along the disk surface as determined from the asymptotic jet properties and the Keplerian rotation of the disk

equation becomes more problematic due to the gradients in the $\Omega_{\mathrm{F}}$-source term.

In summary, only a model including differential rotation $\Omega_{\mathrm{F}}(\Psi)$ may provide a connection between the asymptotic jet, the disk magnetic flux distribution and also the size of the central object. With our model we have presented a reasonable first solution for a self-consistent treatment.

\subsection{Application to the M 87 jet}

The jet of M 87 shows superluminal motion clearly indicating a highly relativistic jet velocity (Biretta et al. 1999). Recent radio observations have been able to resolve the innermost region of the $M 87$ jet formation region with $0.33 \times 0.12$ mas beam resolution (Junor et al. 1999), corresponding to $2.5-7.010^{16} \mathrm{~cm}$. Assuming a central supermassive black hole of $310^{9} M_{\odot}$ (Ford et al. 1994), this is equivalent to about $30 R_{\mathrm{S}}$ ! The derived jet full opening angle is $60^{\circ}$ up to a distance of $0.04 \mathrm{pc}$ from the source with a "strong collimation" occurring afterwards (Junor et al. 1999).

We now apply our two-dimensional jet model to these observations and compare the geometrical scales. Such a comparison is not possible for e.g. self-similar models. From the observed radio profile resolving the inner M 87 jet (see Fig. 1 in Junor et al. 1999), we deduce a jet radius of about 120 Schwarzschild radii. With this, the first important conclusion is that the ratio of jet radius to light cylinder radius must be definitely less than the value of 100 which is usually assumed in the literature. A number value of $3-10$ seems to be much more likely. Numerical models of two-dimensional general relativistic magnetic jets fitting in this picture were calculated by Fendt (1997a). These solutions, however, do not take into account the differential rotation $\Omega_{\mathrm{F}}(\Psi)$.

Junor et al. (1999) claim that the M 87 jet radius in the region "where the jet is first formed cannot be larger than" their resolution of $30 R_{\mathrm{S}}$. Our conclusion is that the expansion rate is limited in both directions. The new radio observations give a minimum value of 3 . Theoretical arguments limit the expansion rate to the 
value of about 20 , since the jet mass flow must originate outside the marginally stable orbit which is located at $3-6 R_{\mathrm{S}}$. Clearly, if the jet radius is really as small as observed in $\mathrm{M} 87$, general relativistic effects may vary the field structure in the jet formation region.

From our model solutions, we derive a light cylinder radius of the M 87 jet of about $50 R_{\mathrm{S}}$. The value derived from Eq. (14) differs from that by a factor of two, but is biased by the unknown size of the disk radius $x_{\text {disk }}$. This parameter, however, does not affect the global solution. Considering the standard relativistic MHD theory, nothing special is happening at the light cylinder. For a highly magnetized plasma wind the light surface corresponds to the usual Alfvén surface which itself does not affect the flow of matter. Hence, the light cylinder is un-observable.

Also the opening angle in our numerical solution is larger than the observed value by a factor of two. This cannot be due to projection effects since any inclination between jet axis and the line of sight will increase the observed opening angle. We hypothesize that a numerical model with a steeper profile for the iso-rotation parameter will give a smaller jet opening angle comparable to the observed data. This is not surprising, since the jet footpoint anchored in a Keplerian disk rotates faster than in our model. Nevertheless, comparing the collimation distance observed in the M 87 jet and assuming a similar ratio of jet radius to light cylinder radius as in our model with $h=0.5$, we find good agreement. The collimation distance is $2 R_{0}$.

In summary, we conclude that the example of the M 87 jet gives clear indication that the light cylinder of AGN jets might not be as large as previously thought. Although our model does not fit the observed geometrical properties of the inner M 87 jet perfectly, we find in general a close compatibility.

\section{Conclusions}

We have investigated the two-dimensional magnetic field distribution in collimating, relativistic jets. The structure of the axisymmetric magnetic flux surfaces is calculated by solving the relativistic force-free Grad-Shafranov equation numerically. In relativistic MHD, electric fields become important in difference to Newtonian MHD. The simplifying assumption of the force-free limit has been applied as relativistic jets must be highly magnetized.

The central point of our paper is the consideration of differential rotation of the foot points of the field lines, i.e. a variation of the iso-rotation parameter $\Omega_{\mathrm{F}}(\Psi)$. The underlying model is that of a magnetic jet anchored in an accretion disk. Two main problems had to be solved in order to calculate a two-dimensional field distribution: a) to determine the a priori unknown location of the light surface, b) the proper treatment of the regularity condition along that light surface. The light surface is the force-free equivalent of the Alfvén surface and provides a singularity in the Grad-Shafranov equation. We summarize our results as follows.
(1) We find numerical solutions for the twodimensional magnetic flux distribution connecting the asymptotic cylindrical jet with a differentially rotating disk. In our example solutions the asymptotic jet radius is about 2.5 times the asymptotic light cylinder radii. This is the first truly two-dimensional relativistic solution for a jet magnetosphere including differential rotation of the iso-rotation parameter $\Omega_{\mathrm{F}}(\Psi)$. The physical solution, being characterized by a smooth transition across the light surface, is unique for a certain parameter choice for the rotation law $\Omega_{\mathrm{F}}$.

(2) The half opening angle of the numerical jet solution is about 60 degrees. Cylindrical collimation is achieved already after a distance of 1-2 asymptotic light cylinder radii along the jet axis. Differential rotation decreases the jet opening angle, but increases the distance from the jet origin where collimation is achieved. The "jet expansion rate", defined as the ratio of the asymptotic jet radius to the jet radius at the jet origin, is about 10 .

(3) From the analytical treatment of the regularity condition along the asymptotic branches of the light surface we derive a general estimate for the jet opening angle. We find that the jet half opening angle is larger than $45^{\circ}$ and increases for a steeper profile of the differential rotation $\Omega_{\mathrm{F}}$.

(4) Our two-dimensional ansatz, in combination with the treatment of differential rotation, allows for a connection of the asymptotic jet solution with the accretion disk. Certain disk properties can be deduced from the asymptotic jet parameters. Examples are the disk toroidal magnetic field distribution, with a maximum at half of the disk radius and the angular momentum flux per unit time and unit radius. This is interesting as a boundary condition for accretion disk models. We find that most of the angular momentum is lost in the outer part of the disk.

(5) Application of our model to the M 87 jet gives good agreement qualitatively. From our numerical solution we derive an asymptotic light cylinder of the M 87 jet of about 50 Schwarzschild radii. Collimation of the jet would be achieved after a distance of two asymptotic light cylinder radii from the source. This value is comparable with the observations, however, the opening angle in our model is larger by a factor of two.

In summary, we have presented the first global twodimensional solutions for a relativistic jet magnetosphere taking into account differential rotation of the jet footpoints. From our jet model we may determine certain physical quantities in the disk that are not possible to observe, as e.g. the angular momentum flux distribution at the disk-jet interface. Comparison with the M 87 jet shows that our model seems to be consistent with the observations, therefore allowing for a derivation of the collimation distance, the light cylinder radius and the jet expansion rate for that example. Clearly, such features as the timedependent ejection of knots and the interaction process between disk, jet and central source cannot be answered by our approach. Time-dependent relativistic MHD 
simulations of the whole "star"-disk-jet system would be necessary, however, such codes are not yet fully developed.

Acknowledgements. Part of this work was initiated when C. F. was holding a guest stipend of the Sonderforschungsbereich (SFB) 328 of the University of Heidelberg. E. M. acknowledges a grant (FE 490/1-1) from the Deutsche Forschungsgemeinschaft (DFG).

\section{Appendix A: Numerical methods}

For the solution of the two-dimensional GS equation we apply the method of finite elements as developed by Camenzind (1987) and Fendt et al. (1995). Differential rotation $\Omega_{\mathrm{F}}(\Psi)$ implies two major complications for the numerical computation. The first one is the fact that position and shape of the light surface $D=0$ is not known a priori. Along the light surface the boundary condition is the regularity condition, which, however, itself depends on the two-dimensional solution $\Psi(x, z)$. The second problem is the GS source term for the differential rotation, containing the gradient of the magnetic flux, $|\nabla \Psi|^{2}$. Compared to the case of rigid rotation, this introduces another (and stronger) non-linearity in the GS equation. Therefore, a fragile numerical convergence process can be expected.

An additional complication is that our grid of finite elements of second order may be inadequate for a calculation of monotonous gradients between the elements if the numerical resolution is too low. However, for appropriate numerical parameters as grid size, element size and iteration step size, we were finally able to overcome these difficulties.

\section{A.1. Determination of the light surface}

Here we discuss the iteration procedure we use to determine the location of the light surface. Because the rotation law $\Omega_{\mathrm{F}}(\Psi)$ is prescribed, the radius where the light surface, $D=0$, intersects the jet boundary, $\Psi=1$, is known,

$x_{\mathrm{L}}(\Psi=1)=1 / \Omega_{\mathrm{F}}(\Psi=1)$.

However, the corresponding position in $z$-direction is not known. Some estimates can be made about shape and inclination of the light surface in the limit of large radii (see Sect. 2.4), but a general solution is not yet known.

We start the iteration procedure calculating the inner solution (defined as the field distribution inside the light surface) with an outer grid boundary at $x=1$ (for comparison see Fig. 1). This choice is equivalent to the light cylinder in the case of rigid rotation. For differential rotation the radius $x=1$ is defined as asymptotic light cylinder (for large $z$ ). For low $z$-values the boundary $x=1$ is located inside the light surface $x_{\mathrm{L}}(\Psi)=1 / \Omega_{\mathrm{F}}(\Psi)$. Along this outer boundary (of the inner solution), we apply a homogeneous Neumann boundary condition. Usually, this implies that the field lines will cross that boundary perpendicularly. However, in our case the homogeneous Neumann boundary condition transforms into the regularity condition if the boundary becomes equivalent to the singular light surface. As shown in Fendt et al. (1995), this transformation applies "automatically" in our finite element code. This is due to the facts that (i) finite element code solves the integrated GS equation and (ii) the boundary integral, which is proportional to $D=1-x^{2} \Omega_{\mathrm{F}}^{2}$, vanishes along the light surface.

With the GS solution of the first iteration step we estimate the deviation of the chosen outer boundary from the true light surface by calculating $D=1-x^{2} \Omega_{\mathrm{F}}^{2}(\Psi)$. For the lowest $z$-value prescribed, we know that $D=$ $1-x^{2} \Omega_{\mathrm{F}}^{2}(\Psi=1)$. Then, the outer grid boundary $(x, z)$ is slowly moved to a larger radius with $\Delta x \sim D(x, z)^{2}$. As a consequence of the different numerical grid, the field distribution will change. The value of $D$ will, however, decrease. This procedure is repeated until $D$ is below a certain limit, $D \simeq 0$. Having obtained the solution inside the light surface, that field distribution is taken as inner boundary condition for the outer solution.

\section{References}

Appl, S., \& Camenzind, M. 1993, A\&A, 274, 699

Beskin, V. S. 1997, Phys. Uspekhi, 40, 659

Biretta, J. A., Sparkes, W. B., \& Maccetto, F. 1999, ApJ, 520, 621

Blandford, R. D., \& Payne, D. G. 1982, MNRAS, 199, 883

Camenzind, M. 1987, A\&A, 184, 341

Camenzind, M. 1990, RvMA, 3, 234

Chiueh, T., Li, Z.-Y., \& Begelman, M. C. 1991, ApJ, 377, 462

Contopoulos, J., \& Lovelace, R. V. E. 1994, ApJ, 429, 139

Contopoulos, J. 1994, ApJ, 432, 508

Contopoulos, J. 1995, ApJ, 446, 67

Fendt, C., Camenzind, M., \& Appl, S. 1995, A\&A, 300, 791

Fendt, C., \& Camenzind, M. 1996, A\&A, 313, 591

Fendt, C. 1997a, A\&A, 319, 1025

Fendt, C. 1997b, A\&A, 323, 999

Ferreira, J. 1997, A\&A, 319, 340

Ford, H. C., et al. 1994, ApJ, 435, L27

Junor, W., Biretta, J. A., \& Livio, M. 1999, Nat, 401, 891

Khanna, R., \& Camenzind, M. 1992, A\&A, 263, 401

Li, Z.-Y. 1993, ApJ, 415, 118

Lovelace, R. V. E., Berk, H. L., \& Contopoulos, J. 1991, ApJ, 379,696

Mirabel, I. F., \& Rodriguez, L. F. 1995, in Superluminal motions in our Galaxy, 17th Texas Symposium on Relativistic Astrophysics and Cosmology, ed. H. Böhringer, G. E. Morfill, \& J. E. Trümper, Annals of the New York Academy of Sciences, New York, 21

Mundt, R., Bührke, T., Solf, J., Ray, T. P., \& Raga, A. C. 1990, A\&A, 232, 37

Nitta, S.-Y. 1994, PASJ, 46, 217

Nitta, S.-Y. 1995, MNRAS, 276, 825

Okamoto, I. 1992, MNRAS, 254, 192

Okamoto, I. 1999, MNRAS, 307, 253

Ray, T. P., Mundt, R., Dyson, J. E., Falle, S. A. E. G., \& Raga, A. C. 1996, ApJ, 468, L103

Pudritz, R. E., \& Norman, C. A. 1983, ApJ, 274, 677

Shibata, K., \& Uchida, Y. 1985, PASJ, 37, 31

Sakurai, T. 1985, A\&A, 152, 121

Zensus, J. A., Cohen, M. H., \& Unwin, S. C. 1995, ApJ, 443, 35 